\title{
Understanding the positive benefit:risk profile of alemtuzumab in relapsing multiple sclerosis: perspectives from the Alemtuzumab Clinical Development Program
}

\author{
This article was published in the following Dove Press journal: \\ Therapeutics and Clinical Risk Management \\ 16 October 2017 \\ Number of times this article has been viewed
}

\section{Eva Havrdova' \\ Jeffrey A Cohen² \\ Dana Horakova' \\ Ivana Kovarova' \\ Eva Meluzinova ${ }^{3}$}

'Department of Neurology and Center of Clinical Neuroscience, First Faculty of Medicine, Charles University and General University Hospital in Prague, Prague, Czech Republic; ${ }^{2}$ Neurological Institute, Cleveland Clinic, Cleveland, $\mathrm{OH}$, USA; ${ }^{3}$ Department of Neurology, Second Faculty of Medicine, Charles University, Motol University Hospital, Prague, Czech Republic
Correspondence: Eva Havrdova Department of Neurology and Center of Clinical Neuroscience, First Faculty of Medicine, Charles University and General University Hospital in Prague, Katerinska

30, Prague 120 00, Czech Republic

Tel +420224966422

Fax +420 22 49I 7907

Email eva.havrdova@gmail.com

\begin{abstract}
The introduction of high-efficacy therapies for relapsing-remitting multiple sclerosis has driven re-evaluation of treatment goals and benefit:risk considerations in treatment choice. In the alemtuzumab Phase II and III clinical trials, patients treated with alemtuzumab $12 \mathrm{mg}$ versus subcutaneous interferon beta-1a demonstrated significantly reduced annualized relapse rates and improved magnetic resonance imaging outcomes, and were significantly more likely to achieve no evidence of disease activity and reduction in brain volume loss. In two of the studies, alemtuzumab-treated patients had a significantly reduced risk of 6-month confirmed disease worsening, compared with subcutaneous interferon beta-1a. Benefits were maintained throughout 5 years, with a majority of patients receiving no alemtuzumab retreatment or other diseasemodifying therapy. Trial results support alemtuzumab's manageable, consistent safety profile in relapsing-remitting multiple sclerosis. Infusion-associated reactions, the most frequent adverse events (AEs), can be minimized by corticosteroid pretreatment, monitoring, and symptomatic management. Other AEs include infections and autoimmune events. Oral anti-herpes prophylaxis should be initiated on the first day of each alemtuzumab treatment course and continued according to local guidelines. Overall cancer risk was lower in the alemtuzumab clinical trials than in a reference population; however, continuing surveillance will determine if alemtuzumab may be associated with certain malignancies such as thyroid papillary carcinoma and melanoma, which are currently identified as potential risks. The post-approval risk management strategy includes a safety monitoring program. Autoimmune AEs (thyroid events, immune thrombocytopenia, nephropathies) can be detected in a timely manner with the monitoring program, which includes physician and patient education about the signs and symptoms, monthly renal and hematologic monitoring, and quarterly thyroid function monitoring for 48 months after the last alemtuzumab course. Education, vigilance by physicians and patients, and monthly laboratory monitoring are recommended to maintain alemtuzumab's positive benefit:risk profile.
\end{abstract}

Keywords: alemtuzumab, annualized relapse rate, autoimmune event, infusion-associated reaction, MRI outcomes, multiple sclerosis, no evidence of disease activity, NEDA, risk mitigation

\section{Introduction}

The introduction of high-efficacy therapies for the treatment of relapsing-remitting multiple sclerosis (RRMS) has shifted expectations for the management of the disease. ${ }^{1-3}$ High-efficacy therapies have stronger treatment effects on relapse rates, disability progression, and brain volume loss (BVL) than classical first-line therapies (eg, interferon beta products or glatiramer acetate), which has led to a re-evaluation of treatment goals 
for multiple sclerosis (MS). ${ }^{1-3}$ For example, the concept of "no evidence of disease activity" (NEDA: no relapses, disability worsening, new gadolinium-enhancing T1 lesions or new/enlarging T2-hyperintense lesions) has become widely accepted as an important treatment objective, albeit one of unclear long-term prognostic value. ${ }^{4-8}$ The higher standards for efficacy prompted by this class of therapies aim to prevent the accumulation of irreversible neurologic damage and reduce the risk of worsening disability. ${ }^{4}$ Furthermore, improvements in function and pre-existing disability are now regarded as feasible goals of therapy. ${ }^{9,10}$

Physicians and patients must agree when it is appropriate to use high-efficacy therapies. A key consideration in choice of therapy is the benefit:risk profile. Traditional first-line agents are directed primarily against inflammation and have demonstrated only mild efficacy in the treatment of MS, with some patients continuing to experience considerable disease activity despite treatment. ${ }^{3}$ In contrast, some disease-modifying therapies (DMTs), including alemtuzumab, have been shown to reduce the rate of BVL as well as the frequency of relapses, ${ }^{3}$ and some (such as alemtuzumab and natalizumab) have facilitated patients' achievement of NEDA. ${ }^{11-14}$ Patients treated with alemtuzumab have also demonstrated confirmed improvements in pre-existing disability. ${ }^{15,16}$

Traditional and high-efficacy agents have distinct adverse event (AE) profiles, which must be considered. While older therapies are associated with AEs and safety risks, the newer therapies have unique safety profiles, which must be weighed against their potential benefits. ${ }^{3}$ The US Food and Drug Administration requires boxed warnings on product information for natalizumab (progressive multifocal leukoencephalopathy) ${ }^{17}$ and alemtuzumab (autoimmune disorders, including thyroid disorders, immune thrombocytopenia [ITP] and nephropathies; and malignancies). ${ }^{18,19}$ Liver toxicity has been reported with natalizumab, ${ }^{20,21}$ and infusionsite and infusion-associated reactions (IARs)/allergic reactions/rashes have been observed with natalizumab, alemtuzumab, and ocrelizumab. 2,11,12,22 The risk of malignancy may be increased with ocrelizumab administration; in controlled trials, malignancies, including breast cancer, occurred more often in patients treated with ocrelizumab than in patients who received subcutaneous interferon beta-1a (SC IFNB-1a) or placebo. ${ }^{2}$ It is clearly important for clinicians to understand the incidence and management of these risks to help patients continue to achieve their treatment goals.

A further key consideration in RRMS management is the risk posed by delaying the initiation of high-efficacy therapies. ${ }^{23}$ Brain and spinal cord damage begins early in the disease course, although it may not be clinically evident, ${ }^{24,25}$ and even a single relapse can produce long-term effects on disability. ${ }^{26}$ Early intervention is thus warranted to prevent continuing disease activity and the accumulation of permanent neurologic damage. ${ }^{27}$

Finally, long-term adherence to therapy can pose an important challenge in MS patient care. Patients often do not persist with therapy or take their medication as instructed, and studies have shown that nonadherence to therapy can lead to diminished health outcomes. ${ }^{28,29}$ One of the major barriers to adherence among MS patients is that most currently available therapies require chronic administration and patients can become fatigued by the need for continual treatment. Additional barriers include administration issues, such as injection anxiety and the need for administration by a trained health care provider, perceived lack of efficacy, and AEs. ${ }^{30}$

Alemtuzumab is a humanized monoclonal antibody that selectively targets the CD52 surface antigen on circulating lymphocytes. It is approved in the European Union for adult patients with RRMS with active disease defined by clinical or imaging features. ${ }^{18}$ In the USA, alemtuzumab is generally indicated for the treatment of patients with relapsing forms of MS who have had an inadequate response to two or more drugs indicated for the treatment of MS, although use as a first-line treatment is not excluded. ${ }^{19}$ Approval was based on the results of two studies - the Phase III Comparison of Alemtuzumab and Rebif ${ }^{\circledR}$ Efficacy in MS (CARE-MS) I and II trials - in which alemtuzumab demonstrated significant clinical and radiologic benefits versus an active comparator, SC IFNB-1a. ${ }^{11,12}$ These benefits were maintained up to 5 years in an extension study ( 2 years of core study plus 3 years of extension); ${ }^{31,32}$ preliminary data from a total of 6 years of treatment were presented at the 32nd Congress of the European Committee for Treatment and Research in Multiple Sclerosis and will be further elaborated in due course. ${ }^{33-35}$ AEs associated with alemtuzumab include IARs, infections, and autoimmune AEs (primarily thyroid disorders and, less frequently, ITP and nephropathies). ${ }^{11,12}$ In the clinical development program, safety monitoring was performed until 48 months after the last dose of alemtuzumab; after a fatal case of ITP occurred during CAMMS223, the monitoring program was modified to include measures for its detection. ${ }^{11,12,36}$ A similar monitoring scheme is recommended in the postmarketing setting. ${ }^{18,19}$ This review discusses benefit:risk considerations for the use of alemtuzumab in the treatment of RRMS.

\section{Alemtuzumab dosing, pharmacokinetics, and mechanism of action}

Alemtuzumab has a dosing schedule distinct from other therapies for MS. Rather than continuous dosing, the recommended 
schedule for alemtuzumab comprises two annual courses consisting of intravenous infusion of $12 \mathrm{mg}$ on each of five consecutive days at baseline ( $60 \mathrm{mg}$ total dose) and on each of three consecutive days ( $36 \mathrm{mg}$ total dose) 12 months later. ${ }^{18,19}$ Additional treatment courses of $12 \mathrm{mg} /$ day on three consecutive days ( $36 \mathrm{mg}$ total dose) may be administered at least 12 months after the prior course, upon documented evidence of MS disease activity.

Serum concentrations of alemtuzumab increase with each consecutive dose within the treatment course, but decrease to low or undetectable levels within $\sim 30$ days after the last infusion. ${ }^{37}$ The elimination half-life of alemtuzumab is $\sim 2$ weeks and is comparable between treatment courses. ${ }^{18}$

The mechanism of action of alemtuzumab is believed to be related to its targeting of CD52, an antigen expressed on the surface of $\mathrm{T}$ and $\mathrm{B}$ lymphocytes. ${ }^{38-40} \mathrm{~T}$-helper type 1 (Th1) cells were long thought to be the main effector T cells responsible for autoimmune inflammation in MS, but studies have disclosed an important pathogenetic role for interleukin (IL)-17-secreting $\mathrm{CD}^{+}{ }^{+} \mathrm{T}$ cells, termed Th17, as well as IL-17-secreting gamma delta T cells, including Th1, Th17, gamma delta, $\mathrm{CD} 8^{+}$, and dysfunctional or impaired regulatory T cells. ${ }^{38-40} \mathrm{~B}$ cells, which are rarely seen in the cerebrospinal fluid (CSF) of healthy subjects, are often present in the CSF of MS patients (albeit in low concentrations). ${ }^{41} \mathrm{~B}$ cells in MS $\mathrm{CSF}$ are primarily of the $\mathrm{CD} 19^{+}, \mathrm{CD} 27^{+}, \mathrm{CD} 138^{-}$phenotype, although mature plasma cells $\left(\mathrm{CD} 19^{-}, \mathrm{CD} 38^{+}, \mathrm{CD} 138^{+}\right)$are also occasionally observed. ${ }^{41} \mathrm{~A}$ higher proportion of $\mathrm{B}$ cells relative to monocytes in $\mathrm{CSF}$ is correlated with more rapid progression of MS. ${ }^{41}$

Analysis of blood samples from patients enrolled in the CARE-MS II clinical trial revealed that alemtuzumab rapidly and selectively depletes both $\mathrm{T}$ and $\mathrm{B}$ lymphocytes (including both $\mathrm{CD} 4^{+}$and $\mathrm{CD} 8^{+} \mathrm{T}$ lymphocytes), ${ }^{39,42}$ with the lowest T- and B-lymphocyte counts observed 1 month after a course of treatment (the earliest posttreatment time point in Phase III studies). ${ }^{18} \mathrm{~B}$ lymphocytes repopulate within about 3 months, whereas $\mathrm{T}$ lymphocytes repopulate more slowly, with the absolute number of both $\mathrm{CD}^{+}$and $\mathrm{CD} 8^{+} \mathrm{T}$ cells remaining decreased up to 12 months. ${ }^{39}$ Further analysis revealed a differential repopulation in T-lymphocyte subsets following alemtuzumab administration. The relative number of $\mathrm{CD}^{+} \mathrm{T}$ cells normalized around Month 3 , whereas $\mathrm{CD} 4^{+}$ $T$ cells remained significantly decreased for up to 24 months, reflecting a selectively delayed reconstitution of only $\mathrm{CD}^{+}$ cells. ${ }^{39}$ There is also a preferential expansion of memory and regulatory $\mathrm{T}$ cells along with $\mathrm{Th} 2$ cells during immune reconstitution, combined with a significant decrease in the percentages of Th1 and Th17 $\mathrm{CD}^{+}$cells, which results in a shift toward a more anti-inflammatory profile. ${ }^{39}$ Reduction in the level of circulating $\mathrm{T}$ and $\mathrm{B}$ cells and subsequent repopulation may also reduce the potential for relapse, which ultimately delays disease progression..$^{39,43-45}$ These changes suggest a rebalancing of the immune system in the absence of continuous administration. ${ }^{43-45}$

In vitro data derived from blood samples obtained from participants in the alemtuzumab Phase II trial also demonstrated that after depletion by alemtuzumab, repopulating $\mathrm{T}$ cells secreted neurotrophic factors that prolonged the life of neurons in an animal model and enhanced oligodendrocyte precursor cell survival, maturation, and myelination. ${ }^{46}$ This observation supports an additional mechanistic hypothesis, termed "neuroprotective autoimmunity". ${ }^{46}$ Further, a recent study demonstrated that regulatory $\mathrm{T}$ cells can directly promote oligodendrocyte progenitor cell differentiation and myelination in vitro, revealing a new, regenerative function of regulatory $\mathrm{T}$ cells in the central nervous system, which is distinct from immunomodulation. ${ }^{47}$ Innate immune cells (such as neutrophils, monocytes, eosinophils, basophils, and natural killer cells), some tissue-resident lymphocytes, and serum immunoglobulin levels are only minimally or transiently affected by alemtuzumab; all these elements contribute to preserving immune defense against infection. . $^{37,44,45,48-50}$

\section{Efficacy of alemtuzumab in RRMS}

The efficacy and safety of alemtuzumab were assessed in three randomized, rater-blinded, active-controlled, headto-head studies: the 3-year, Phase II CAMMS223 study (NCT00050778) ${ }^{36}$ and the 2-year, Phase III CARE-MS studies, which evaluated alemtuzumab $12 \mathrm{mg}$ /day in patients with active RRMS who were treatment naive (CARE-MS I; NCT00530348) $)^{11}$ or had an inadequate response to prior therapy at baseline (CARE-MS II; NCT00548405). ${ }^{12}$ Treatments most commonly used by patients who initiated treatment with alemtuzumab $12 \mathrm{mg} /$ day in CARE-MS II were IFNB-1a (54\%), IFNB-1b (36\%), and glatiramer acetate (34\%); the same therapies were used by similar proportions of patients in the SC IFNB-1a arm of the study. ${ }^{12}$ Coprimary endpoints in CAMMS223 and CARE-MS I and II were reduction in annualized relapse rate and time to 6-month sustained accumulation of disability; ${ }^{11,12,36}$ this latter endpoint is now more commonly described as confirmed disability worsening (CDW). In all three studies, alemtuzumab $12 \mathrm{mg}$ significantly reduced the annualized relapse rate compared with SC IFNB-1a, and alemtuzumab-treated patients in CAMMS223 and CARE-MS II had a significantly reduced risk of 6-month CDW versus SC IFNB-1a. Although the CDW endpoint was not met in CARE-MS I, analysis of 
pooled data from the CAMMS223 and CARE-MS I studies indicated reduced risk of CDW in the treatment-naive population as well. ${ }^{11,51}$ Alemtuzumab-treated patients also had improved magnetic resonance imaging (MRI) outcomes, including significant reductions in BVL, and were significantly more likely to achieve NEDA. ${ }^{11,12}$ Notably, significant improvements in pre-existing disability were observed in alemtuzumab-treated patients compared with SC IFNB-1a in CARE-MS II. ${ }^{12}$

Long-term efficacy of alemtuzumab $12 \mathrm{mg}$ was evaluated in an extension study (NCT00930553), for which 5-year results (2 years in the Phase III studies and 3 years in the extension) have been reported in detail..$^{31,32,52-56}$ In the extension study, additional courses of alemtuzumab were administered as needed at least 12 months after the prior course. By Year 5, most patients had not received alemtuzumab retreatment (CARE-MS I, 68\%; CARE-MS II, 60\%) or another DMT (98\% and 92\%, respectively). ${ }^{54,55}$ Overall, $67 \%$ of patients in CARE-MS I and 54\% of those in CARE-MS II received neither alemtuzumab nor another DMT after their initial two courses of alemtuzumab treatment; ${ }^{31,32}$ yet at 5 years, these patients still experienced continued efficacy on clinical and MRI disease activity measures. ${ }^{52-56}$ A low annualized relapse rate was maintained over Years 3-5 (0.16 and 0.21 in CARE-MS I and II, respectively), and disability, as assessed by change in Expanded Disability Status Scale score, remained stable or improved versus core study baseline to the end of Year 5 (82\% and $77 \%$ of patients in CARE-MS I and II, respectively). ${ }^{15,55,57}$ Further, at Year 5, $33 \%$ of patients in CARE-MS I and $43 \%$ in CARE-MS II achieved 6-month confirmed disability improvement. ${ }^{15,57}$ In a pooled analysis of patients from CARE-MS I and II who continued in the extension study through Year 5 and received only two courses of alemtuzumab ( $\mathrm{N}=445), 84 \%$ had no evidence of CDW and $43 \%$ achieved 6-month confirmed disability improvement; of the latter group, $96 \%$ were free from 6-month CDW. ${ }^{58}$ Consistent with the core studies, most patients receiving alemtuzumab $12 \mathrm{mg}$ were free of MRI activity during Years 3 (CARE-MS I, 72\%; CARE-MS II, $68 \%$ ), 4 (70\% and 70\%, respectively), and 5 (70\% and 68\%, respectively). ${ }^{52,56}$ More than half of alemtuzumab-treated patients achieved annual NEDA in Years 3 (CARE-MS I, $62 \%$; CARE-MS II, 53\%), 4 (60\% and 55\%, respectively), and 5 (62\% and 58\%, respectively). ${ }^{54,55}$ Among the alemtuzumab-treated CARE-MS I patients who achieved NEDA in Year 2 and received no further treatment following the initial two courses of alemtuzumab, $>60 \%$ attained annual NEDA sustained from Years 2 to $5 .{ }^{55}$ Among the equivalent cohort in CARE-MS II, nearly half attained annual NEDA sustained from Years 2 to $5 .{ }^{54}$

Median yearly BVL decreased progressively in CARE-MS I, remaining low in Years 3-5 (Year 1: $-0.59 \%$, Year 2: $-0.25 \%$, Year 3: $-0.19 \%$, Year 4: $-0.15 \%$, Year 5: $-0.20 \%$ ). Likewise, in CARE-MS II, median yearly BVL slowed and remained low in Years 3-5 (Year 1: $-0.48 \%$, Year 2: $-0.22 \%$, Year 3: $-0.10 \%$, Year 4: $-0.19 \%$, Year 5: $-0.07 \%){ }^{53}$

Patient-reported outcome data from the CARE-MS studies further support the efficacy of alemtuzumab in patients who had an inadequate response to prior therapy. Improvements from baseline in physical, mental, and emotional aspects of quality of life observed in the first year of alemtuzumab treatment were maintained in the extension study through Year 4. ${ }^{59,60}$ Significantly greater improvements in these quality-of-life measures were observed in alemtuzumabtreated patients who experienced improvement in pre-existing disability, compared with those who did not. ${ }^{61}$

\section{Overview of safety with alemtuzumab}

Results of the CARE-MS core and long-term extension studies support a consistent and manageable safety profile for alemtuzumab in patients with RRMS. The most frequent AEs were IARs, which occurred most commonly during the first course of infusions and decreased thereafter. ${ }^{11,12,62}$ Other notable AEs included infections, which were serious in only a small percentage of patients, and autoimmune AEs (thyroid disorders, ITP, and nephropathies), as shown in Figure 1A and B. More detailed information on each of these events, along with strategies designed to mitigate them, is provided in the following sections.

\section{Improving benefit:risk with alemtuzumab: detection and management of AEs}

The clinical development program for alemtuzumab included a number of steps to mitigate the potential risks associated with treatment. These were prophylactic measures, laboratory testing and monitoring, patient screenings, and provision of instructional and educational aids. The sections that follow describe these risk mitigation measures, the incidence of AEs in patients receiving alemtuzumab $12 \mathrm{mg}$ in clinical trials, and information about how these AEs were managed.

\section{Infusion-associated reactions}

IARs are common with alemtuzumab ${ }^{11,12,62}$ and are believed to be attributable to cytokine-release syndrome involving target cell lysis and further recruitment of inflammatory cells. ${ }^{18,63,64}$ In the clinical trials, an IAR was defined as any AE occurring 


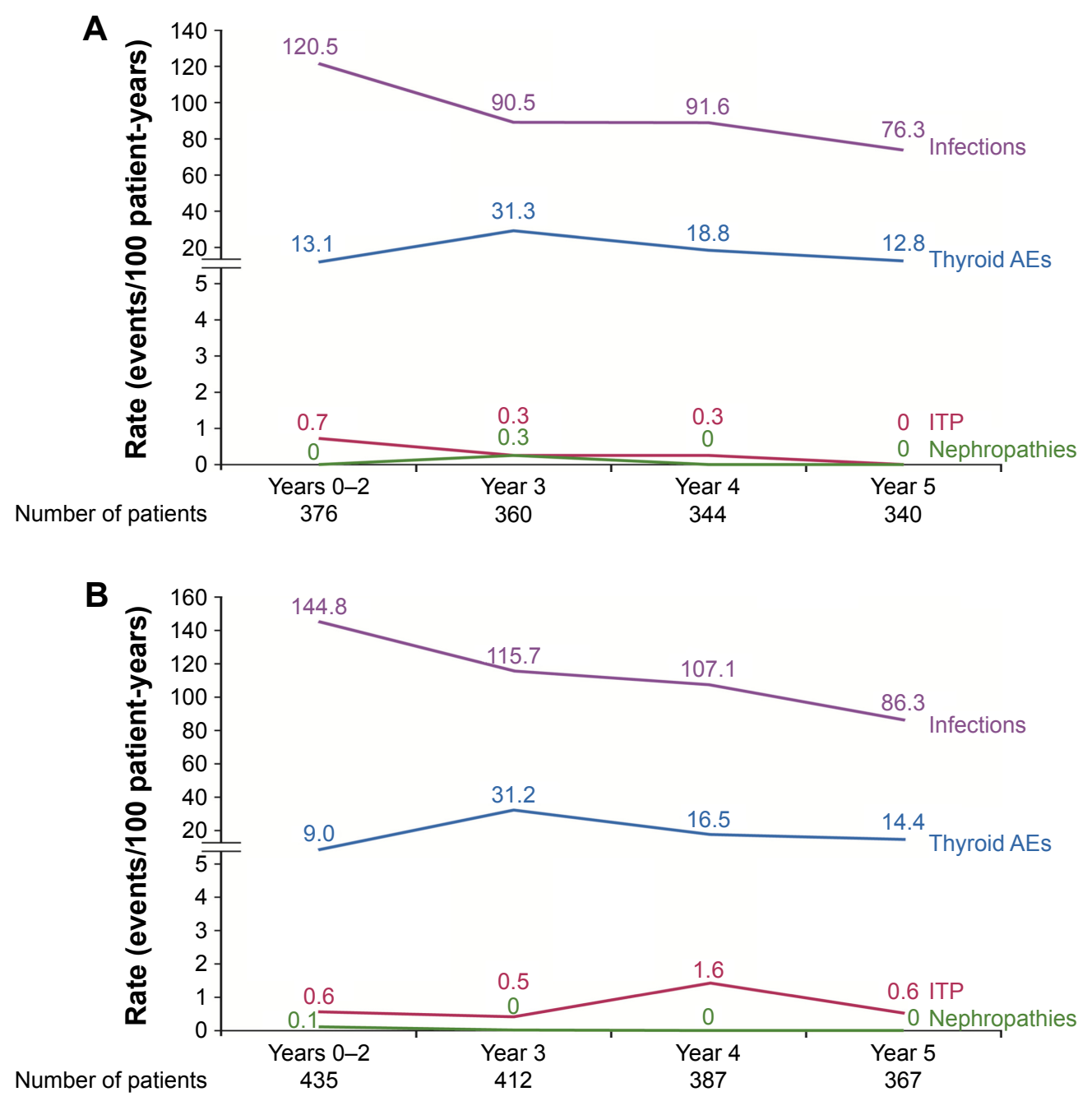

Figure I Rate (events/I00 patient-years) of notable AEs by year over 5 years in patients receiving alemtuzumab I2 mg in the (A) CARE-MS I and (B) CARE-MS II core (Years 0-2) and extension (Years 3-5) studies. ${ }^{15,57,76}$

Abbreviations: AEs, adverse events; CARE-MS, Comparison of Alemtuzumab and Rebif ${ }^{\circledast}$ Efficacy in MS; ITP, immune thrombocytopenia.

during or within 24 hours after alemtuzumab infusion. ${ }^{11,12}$ IARs were most frequent with the first alemtuzumab course, but decreased for the second course, and in subsequent courses in patients who required retreatment (Course 1: 84.7\%; Course 2: 68.6\%; Course 3: 64.6\%; Course 4: 62.8\%; Course 5: 45.5\%). ${ }^{62}$ Most IARs were mild or moderate, and the incidence of serious IARs over Years 0-5 was low (3.2\%). The most common types of IARs were headache, rash, and pyrexia. IARs were minimized by pretreatment or management with medication, monitoring, and infusion adjustment (Figure 2) ${ }^{62}$ Preinfusion measures included pretreatment of all patients with methylprednisolone $1 \mathrm{~g}$ administered intravenously (IV) immediately prior to infusion on each of the first 3 days of any treatment course. During alemtuzumab infusion, patients were closely monitored and were given symptomatic treatment for common IARs (eg, headache, rash, pyrexia, nausea, and flushing). The infusion could be slowed, interrupted, or discontinued in the event of an IAR. ${ }^{65,66}$ Prolonging treatment courses by up to 5 additional days did not appear to increase the incidence of serious or moderate/severe IARs, had no impact on lymphocyte depletion, and did not appear to affect efficacy outcomes. ${ }^{67}$

Consistent with procedures used in the clinical trials, alemtuzumab should be administered in a setting with appropriate equipment and personnel to manage anaphylaxis or serious IARs. ${ }^{18,19}$ The approved product label advises premedication with corticosteroids immediately prior to alemtuzumab infusion for the first 3 days of any treatment course. ${ }^{18,19}$ However, it was the authors' clinical experience during the original trials that when $1 \mathrm{~g}$ IV methylprednisolone was administered for only 3 days, patients had a higher rate of IARs, especially skin exanthems, at Days 4 and 5 . 


\begin{tabular}{|l|}
\hline \multicolumn{1}{|c|}{ Before infusion } \\
\hline Premedication \\
- IAR prophylaxis with $1 \mathrm{~g} \mathrm{IV}$ \\
methylprednisolone immediately \\
prior to infusion on each of the \\
first 3 days of any treatment course \\
- Pretreatment with antipyretics, \\
antihistamines, histamine \\
$\mathrm{H}_{2}$-receptor blockers, and/or \\
antiemetics was also permitted \\
at the investigator's discretion \\
to potentially minimize IARs \\
\hline
\end{tabular}

\begin{tabular}{|c|}
\hline During infusion \\
\hline $\begin{array}{l}\text { Patient observation } \\
\text { - Hourly vital signs } \\
\text { - Adequate hydration }\end{array}$ \\
\hline $\begin{array}{l}\text { Symptomatic treatment for common IARs } \\
\text { - Urticarial rash } \\
\text { - Nonselective antihistamine (IV for rapid onset; some } \\
\text { administered intramuscularly) } \\
\text { - Headache, myalgia, rigors } \\
\text { - Analgesic (eg, acetaminophen) } \\
\text { - Narcotic analgesia } \\
\text { - Triptan (for more persistent or migrainous headache) }\end{array}$ \\
\hline $\begin{array}{l}\text { Infusion rate adjustment or interruption } \\
\text { - Infusions were typically administered over a period of } \geq 4 \text { hours; } \\
\text { maximum infusion period was }<8 \text { hours } \\
\text { - Infusions were interrupted and resumed at a lower rate, or } \\
\text { discontinued, in a minority of patients }\end{array}$ \\
\hline
\end{tabular}

After infusion

\section{Monitoring}

- Continued monitoring for

$1-2$ hours post-infusion for potential IARs

Optional prescriptions (investigator's discretion)

- Antihistamine, antipyretics

- Antiemetics for nausea

- Analgesics for pain

- Sleep aids

Figure 2 IAR management strategies in the alemtuzumab clinical trials. Reproduced with permission from Mayer L, Casady L, Clayton G, et al. Management of infusionassociated reactions in alemtuzumab-treated relapsing-remitting multiple sclerosis patients (P880). Paper presented at: Joint Americas Committee for Treatment and Research in Multiple Sclerosis (ACTRIMS) - European Committee for Treatment and Research in Multiple Sclerosis (ECTRIMS) Meeting; September I0-13, 2014; Boston, MA, USA. ${ }^{95}$ Abbreviations: IARs, infusion-associated reactions; IV, intravenous.

After alemtuzumab was approved, the authors instead administered $500 \mathrm{mg}$ IV methylprednisolone for 5 days, after which the occurrence of IARs on Days 4 and 5 appeared to be lower. In the authors' opinion, therefore, premedication with methylprednisolone $500 \mathrm{mg}$ IV daily on 5 consecutive days is preferable to the standard recommendation of $1 \mathrm{~g}$ IV for 3 days to prevent the possible occurrence of an IAR on Day 4 or 5 . The authors also highly endorse the label recommendation for pretreatment with antihistamines and/or antipyretics. Despite pretreatment, IARs may still occur. The patient's vital signs should be monitored before and periodically during the infusion. Symptomatic treatment for IARs should be provided as needed (eg, antipyretics, antihistamines, $\mathrm{H}_{2}$ antagonists, fluids). In the event of a serious IAR, physicians may consider immediately discontinuing the infusion. Patients should be monitored for 2 hours after the end of the infusion and should be advised that IARs may occur beyond the 2-hour monitoring period. It is noteworthy that no patients experienced anaphylaxis in the Phase III clinical trials. ${ }^{11,12}$

\section{Infection}

Infection was observed in both the alemtuzumab $12 \mathrm{mg}$ and SC IFNB-1a treatment arms during the CARE-MS core studies (CARE-MS I: $67 \%$ and $45 \%$ for alemtuzumab and SC IFNB-1a, respectively; CARE-MS II: $77 \%$ and $66 \%$, respectively). ${ }^{11,12}$ Infections were mostly mild or moderate (Grade 1 or 2) in severity, and the incidence of serious infection was low (CARE-MS I: $2 \%$ and $1 \%$ for alemtuzumab and SC IFNB-1a, respectively; CARE-MS II: $4 \%$ and $1 \%$, respectively). The most common infections reported with alemtuzumab $12 \mathrm{mg}$ in the core studies were nasopharyngitis (CARE-MS I: 20\%; CARE-MS II: 29\%), urinary tract infection (CARE-MS I: 17\%; CARE-MS II: 21\%), upper respiratory tract infection (CARE-MS I: 15\%; CARE-MS II: 16\%), and herpes viral infections (CARE-MS I: 16\%; CARE-MS II: 16\%). ${ }^{11,12}$ Serious infections included pneumonia, appendicitis, herpes zoster, gastroenteritis, pulmonary tuberculosis, disseminated tuberculosis, and meningitis herpes (all $\leq 1 \%$ ). The rate of infection in alemtuzumab-treated patients was highest during the core studies and declined thereafter over a total of 5 years (Figure 1); the rate of serious infections in Year 5 was 0.59 events per 100 patient-years in CARE-MS I and 2.8 events per 100 patient-years for CARE-MS II. ${ }^{15,57}$

Following a protocol amendment during the core studies, alemtuzumab-treated patients were administered acyclovir $200 \mathrm{mg}$ twice daily, starting on the first day of each treatment course and continuing for 28 days after the last day, for prophylaxis against herpes infection. Acyclovir prophylaxis was also administered with each treatment course during the extension. ${ }^{68}$ In the core studies, acyclovir reduced the incidence of herpetic infection in the month after treatment (Course 1, $0.5 \%$ with acyclovir versus $4.6 \%$ without; Course $2,0.8 \%$ versus $2.6 \%$ ). The incidence of herpes simplex (including herpes simplex, oral herpes, genital herpes, and herpes simplex ophthalmic) was $13.3 \%$ overall and decreased over time (Year 1, 9.0\%; Year 2, 5.4\%; Year 3, $3.1 \%$; Year 4, 2.5\%). The incidence of herpes zoster (including multidermatomal) was $9.2 \%$ overall. ${ }^{68}$

On the basis of experience in the clinical development program in RRMS, several measures are recommended to minimize the risk of herpes infection in patients treated 
with alemtuzumab. ${ }^{18,19}$ Prophylaxis with an oral anti-herpes agent should be initiated on the first day of each alemtuzumab treatment course. The European Summary of Product Characteristics advises continuing oral anti-herpes prophylaxis for a minimum of 1 month following treatment with alemtuzumab, ${ }^{18}$ whereas in the USA, the approved product information states that prophylaxis should be continued for a minimum of 2 months following treatment with alemtuzumab or until the $\mathrm{CD}^{+}$lymphocyte count reaches at least 200 cells/ $\mu \mathrm{L}$ (whichever occurs later). ${ }^{19}$ Patients should have completed necessary immunizations at least 6 weeks before treatment. Live viral vaccines should not be administered to MS patients who have recently received a course of alemtuzumab. Patients with a history negative for chickenpox or vaccination against varicella zoster virus (VZV) should be tested for antibodies to VZV before initiation of treatment. VZV vaccination of antibody-negative patients should be considered prior to treatment initiation, and treatment should be postponed for 6 weeks following vaccination. Delaying the initiation of treatment should be considered for patients with active infection until the infection is fully controlled.

Opportunistic infections following alemtuzumab treatment in the postmarketing setting have been reported. Pharmacovigilance data through February 2017 on 13,000 patients treated with alemtuzumab for MS showed that the most commonly observed opportunistic infections were Listeria monocytogenes and cytomegalovirus (CMV), with estimated frequencies of $0.26 \%$ and $0.13 \%$, respectively. There have been no medically confirmed reports of progressive multifocal leukoencephalopathy attributed to alemtuzumab to date. ${ }^{69}$ Evaluation of postmarketing safety reports from multiple sources is continually performed to increase understanding of the benefit:risk profile of alemtuzumab, to identify possible new safety signals, and to ensure that alemtuzumab labeling is updated to incorporate new information when clinically relevant. However, postmarketing frequencies are not directly comparable to clinical trial incidences because of differences in ascertainment methodology and follow-up duration. It should also be noted that postmarketing reports may include nonserious cases, most cases cannot be confirmed, diagnosis may be inaccurate, and/or there may be duplicate cases or underreporting.

A case study and literature review of Listeria infection in alemtuzumab-treated MS patients documents at least 16 cases of listeriosis, of which at least one was fatal. ${ }^{70}$ Severe neurologic sequelae were reported in one patient. ${ }^{71}$ Listeria infection is associated with food sources; indeed, one of the case reports described recent exposure to foods including smoked sausage and soft cheese. ${ }^{70}$ The alemtuzumab product label recommends that patients avoid or adequately heat foods that may be sources of Listeria, such as raw fish and meat, unpasteurized dairy products, and pasteurized products susceptible to Listeria contamination during the production process. ${ }^{18}$ While current guidelines advise that patients should avoid high-risk foods for at least 1 month after alemtuzumab administration, recent reports suggest (in light of Listeria's observed incubation range of $1-70$ days $)^{72}$ that patients avoid dietary risk factors for several weeks before the scheduled alemtuzumab infusion. ${ }^{18,70}$

Several case studies from routine clinical experience have described additional opportunistic infections following alemtuzumab treatment. Two patients developed CMV $\leq 1$ month after the initial 5-day course; both recovered with treatment. ${ }^{73}$ In a group of 66 patients treated with a total of 100 courses of alemtuzumab throughout 1 year at a center in Germany, three developed acute acalculous cholecystitis, probably secondary to infection. ${ }^{74}$ All three patients made a prompt recovery with antibiotic treatment. Finally, a rare pathogen, Nocardia beijingensis, was isolated from a patient in the southern USA, in whom sepsis secondary to pneumonia was confirmed 6 weeks after completion of alemtuzumab Course 1; the patient responded well to treatment. ${ }^{75}$

\section{Autoimmune AEs}

Treatment with alemtuzumab increases the risk of autoimmunemediated conditions, including thyroid disorders, ITP, other cytopenias (including neutropenia, hemolytic anemia, and pancytopenia), and nephropathies. ${ }^{11,12}$ The clinical development program for alemtuzumab included a number of protocol-defined laboratory monitoring measures that allow early detection and management of autoimmune AEs.

\section{Thyroid AEs}

Thyroid AEs were the most common autoimmune AEs observed in the alemtuzumab clinical trials. ${ }^{11,12,36}$ In the clinical development program, monitoring for thyroid AEs included quarterly testing of thyroid-stimulating hormone (TSH) levels and, if TSH results were abnormal, of free T3 and T4; thyroid autoantibodies at the investigator's discretion; and referral for consultation with an endocrinologist. Overall, 39\% $(n=317)$ of alemtuzumab-treated patients in the CARE-MS studies had thyroid AEs over 5 years. ${ }^{76}$ Most thyroid AEs were mild or moderate (Grade 1 or 2) in severity; $4.4 \%$ of patients had serious thyroid events. ${ }^{76}$ Most events $(81 \%)$ occurred in the first 2 years after the most recent alemtuzumab dose (Figure 1). ${ }^{76}$ The most frequently 
Table I Rates of thyroid AEs by yeara (alemtuzumab $12 \mathrm{mg}$ )

\begin{tabular}{|c|c|c|c|c|}
\hline $\begin{array}{l}\text { System organ class } \\
\text { preferred term }\end{array}$ & $\begin{array}{l}\text { Years 0-2 } \\
(n=8 \mid I)\end{array}$ & $\begin{array}{l}\text { Year } 3 \\
(n=772)^{b}\end{array}$ & $\begin{array}{l}\text { Year } 4 \\
(n=731)\end{array}$ & $\begin{array}{l}\text { Year } 5 \\
(n=707)\end{array}$ \\
\hline Patient-years of follow-up & $\mathrm{I}, 6 \mathrm{I} 2.8$ & 726.1 & 717.0 & 698.5 \\
\hline $\begin{array}{l}\text { Rate (events/ I00 patient- } \\
\text { years) }\end{array}$ & 10.9 & 31.3 & 17.6 & 13.6 \\
\hline Hypothyroidismc & 2.2 & 5.5 & 2.8 & 1.7 \\
\hline Hyperthyroidism & 1.6 & 6.9 & 3.8 & 3.0 \\
\hline Graves' disease & 1.2 & 6.3 & 2.6 & 1.7 \\
\hline Goiter $^{d}$ & 0.7 & 0.8 & 1.0 & 0.7 \\
\hline Autoimmune thyroiditis & 0.7 & 1.5 & 1.1 & 0.6 \\
\hline Thyroiditis $^{\mathrm{e}}$ & 0.3 & 0.6 & 0.3 & 0.3 \\
\hline Thyroid disorder & 0 & 0.4 & 0 & 0.1 \\
\hline Thyroid mass & 0.1 & 0 & 0 & 0 \\
\hline Thyrotoxic crisis & 0.1 & 0 & 0 & 0 \\
\hline Endocrine ophthalmopathy & 0.1 & 0.8 & 0.7 & 1.0 \\
\hline Exophthalmos & 0 & 0 & 0 & 0.3 \\
\hline Laboratory abnormalities & 3.7 & 7.6 & 3.1 & 3.0 \\
\hline Thyroidectomy & 0.1 & 0.7 & 2.1 & 1.0 \\
\hline
\end{tabular}

Notes: aYears are defined by calendar time from first alemtuzumab dose. 'Includes the extension study safety population and patients who did not enter the extension study but were evaluated for AEs for a short time in Year 3 after the initial 2-year period. 'Does not include the preferred term primary hypothyroidism (one event in Year 2). 'Does not include the preferred terms hypothyroidic goiter (one event

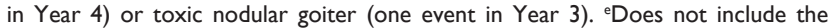
preferred terms thyroiditis subacute (one event each in Years I and 2) or thyroiditis chronic (one event in Year 5). Reproduced with permission from Senior PA, Arnold DL, Cohen JA, et al. Incidence and timing of thyroid adverse events in patients with RRMS treated with alemtuzumab through 5 years of the CARE-MS Studies. Neurology. 2016;86(I6 suppl):P2.086. ${ }^{76}$

Abbreviation: AEs, adverse events.

reported thyroid AEs were hypothyroidism, hyperthyroidism, and Graves' disease (Table 1). Rates (events per 100 patientyears) of the most frequently reported serious thyroid AEs during Years 1-5 were Graves' disease (2.8), hyperthyroidism (1.1), hypothyroidism (0.4), and goiter (0.2). Serious thyroid AEs that occurred in Year $5(\mathrm{n}=7)$ were Graves' disease (0.6), hyperthyroidism (0.1), autoimmune thyroiditis $(0.1)$, and endocrine ophthalmopathy (0.1). Consistent with the pattern observed in long-term follow-up of the Phase II CAMMS223 patients, the proportion of patients with thyroid events peaked at Year 3 and subsequently declined (Year 1: 5.7\%; Year 2: 10.7\%; Year 3: 20.9\%; Year 4: 12.6\%; Year 5: 10.0\%). ${ }^{76}$

The majority of alemtuzumab-treated patients (60.9\%) did not experience thyroid AEs up to Year 4 after the last alemtuzumab dose. ${ }^{76}$ Of the $39 \%$ of patients who experienced a thyroid AE, 63 (19.9\%) did not require treatment for thyroid AEs. Among those who received treatment in Years 0-5, most were successfully managed with first-line therapies, including conventional oral medications (79.8\%) and iodine ablation (7.6\%); 8.2\% underwent thyroidectomy, reflecting the local treatment protocol. ${ }^{76}$ Safety monitoring and education allowed for early detection and effective management of thyroid AEs in the clinical trials. In clinical practice, risk mitigation for thyroid AEs should include thyroid function tests (eg, TSH) before initiation of alemtuzumab and every 3 months thereafter, until 48 months after the last infusion. ${ }^{18,19}$

\section{Immune thrombocytopenia}

As of December 2014, the overall incidence of medically confirmed ITP was $2.3 \%$ in alemtuzumab-treated patients in the clinical development program (corresponding to a rate of 0.44 events $/ 100$-patient years). ${ }^{77}$ Alemtuzumab-associated ITP is a unique form of ITP generally characterized by delayed presentation after drug exposure, responsiveness to conventional ITP therapies, and prolonged remission. Alemtuzumab-associated ITP has been compared to pediatric ITP, which generally follows a limited course, in contrast to primary ITP in adults, in which durable remission is rarely achieved ( $\sim 10 \%$ with first-line therapy). ${ }^{78} \mathrm{~A}$ transient decrease in platelets has occasionally been observed during alemtuzumab infusion and can be differentiated from ITP by a rapid return to normal thrombocyte values. ${ }^{79,80}$

A monitoring program for ITP was implemented throughout the Phase III and extension studies in response to initial reports of ITP in three patients (fatal in one case) receiving alemtuzumab $24 \mathrm{mg}$ in the Phase II CAMMS223 study (the $24 \mathrm{mg}$ dose is not used in clinical practice). ${ }^{78}$ The program included establishment of a protocol definition of ITP, investigator education about ITP, and patient education regarding signs and symptoms of ITP. The protocol definition of ITP was based upon the diagnostic threshold (platelet count $<100 \times 10^{9} / \mathrm{L}$ ) outlined by an international working group: ${ }^{81}$ either a confirmed platelet count $\geq 50 \times 10^{9} / \mathrm{L}$, but $<100 \times 10^{9} / \mathrm{L}$ on $\geq 2$ consecutive occasions over a period of at least 1 month, or a confirmed platelet count $<50 \times 10^{9} / \mathrm{L}$ without clumping documented on $\geq 2$ consecutive occasions over any period of time. Monthly complete blood counts (CBCs) as well as monthly symptom surveys, at 2-week intervals from $\mathrm{CBC}$, were performed. ITP events were reviewed and confirmed if there was no alternative etiology and the event met the platelet criterion of $<100 \times 10^{9} / \mathrm{L}$, consistent with the consensus definition of primary ITP. ${ }^{77}$ When ITP was diagnosed, alemtuzumab treatment was discontinued and patients were referred for hematologic consultation. Alemtuzumab-treated patients who develop ITP are followed for a minimum of 48 months from ITP diagnosis. Similar to the ITP monitoring program implemented in clinical trials, the prescribing information for alemtuzumab recommends that $\mathrm{CBC}$ with differential be obtained prior to initiation of treatment and at monthly intervals until 48 months after the last infusion. ${ }^{18,19}$ Monthly symptom surveys (at 2-week 
intervals after $\mathrm{CBC}$ ) should also be performed. If ITP is suspected, a CBC should be obtained immediately, as confirmed ITP requires prompt medical intervention. Patients should be counseled to remain alert for symptoms of ITP, which may include easy bruising, petechiae, spontaneous mucocutaneous bleeding (eg, epistaxis, hemoptysis), or heavier-than-normal or irregular menstrual bleeding. In the context of alemtuzumab treatment, hemoptysis may be a sign of alveolar hemorrhage associated with anti-glomerular basement membrane (anti-GBM) disease; differential diagnosis is necessary to rule out Goodpasture syndrome.

Of the 34 patients $(2.3 \%)$ who developed ITP in the clinical development program (21 of whom had received the $12 \mathrm{mg}$ dose of alemtuzumab), the majority had received only the original two courses of alemtuzumab (one course, $\mathrm{n}=3$; two courses, $\mathrm{n}=21$; three courses, $\mathrm{n}=9$; four courses, $\mathrm{n}=1) .{ }^{77}$ All ITP events after the fatal index case were detected through the risk monitoring program; $\sim 80 \%$ were detected through monthly blood monitoring and $20 \%$ through patient recognition of signs and symptoms. All but three patients received treatment for ITP (the one fatal index case, which was diagnosed postmortem, and two patients who had spontaneous resolution). All patients who received treatment for ITP had a complete response (defined as an increase in the platelet count to $\geq 10 \times 10^{10} / \mathrm{L}$ in the absence of bleeding and no ongoing therapy). Twenty-two of 31 patients recovered with first-line therapy (ie, IV immunoglobulin, corticosteroids, and/ or platelet transfusion) and seven had a complete response to second-line therapy (rituximab or, in the case of one patient, splenectomy).$^{77}$ One patient experienced an ITP recurrence. The patient was treated with alemtuzumab and, 16 months after the last infusion, experienced an episode of ITP, which resolved without treatment. Seven years later, while on fingolimod treatment, the patient had a recurrence of ITP, which was treated with IV corticosteroids. ${ }^{77}$ In all other patients treated for ITP, remission was sustained. Evidence from clinical trials supports the value of patient education regarding signs and symptoms of ITP and of adherence to monitoring requirements to facilitate its early detection and effective management.

\section{Nephropathies}

In clinical trials, renal function was monitored by means of quarterly serum creatinine levels. Following identification of the first nephropathy case, which occurred outside the clinical development program, monitoring for kidney injury was modified to include monthly serum creatinine and urinalysis. An educational component of the monitoring program ensured that patients and physicians were made aware of the possible signs and symptoms of renal dysfunction. A total of four cases of nephropathy (total incidence, $0.27 \%$ ) were reported during the alemtuzumab clinical development program: one patient each in CAMMS223 and CARE-MS II and two in the extension study. Of the four cases, one was anti-GBM disease, two were membranous glomerulonephritis (MGN), and one was an unconfirmed case of MGN with seropositivity for anti-GBM antibody. The case of anti-GBM disease was identified on investigation after the patient's serum creatinine was found to be three times the upper limit of normal. The two MGN cases were initially detected on the basis of hematuria/proteinuria and on a patient report of leg edema, and were confirmed after biopsy. For the fourth case, biopsy revealed weak evidence for mild, stage $1 \mathrm{MGN}$, with no evidence of anti-GBM disease, although the patient was positive for anti-GBM antibodies. All four patients responded to timely medical treatment, and none required dialysis or renal transplant. ${ }^{82}$

Clinical manifestations of nephropathy may include elevated serum creatinine levels, hematuria, and/or proteinuria; thus, the prescribing information for alemtuzumab advises that both serum creatinine levels and microscopic urinalysis with cell counts must be obtained prior to initiation of alemtuzumab treatment and at monthly intervals until 48 months after the last infusion. ${ }^{18,19}$ Because urinalysis with microscopy is time consuming and requires skilled personnel, some clinics use simpler methods of urinalysis for practical reasons. Hemoptysis was not observed in clinical trials; however, alveolar hemorrhage manifesting as hemoptysis may occur with anti-GBM disease or be indicative of ITP, and an appropriate differential diagnosis is warranted. Clinicians should also remind patients to watch for symptoms (eg, hematuria, edema). Because anti-GBM disease may lead to permanent renal failure and can be life-threatening if left untreated, testing should be performed based on any clinical findings suggestive of nephropathies. Clinical trial evidence suggests that early detection and treatment of nephropathies is likely to improve renal outcomes. ${ }^{82}$

\section{Other potential autoimmune events}

In a case report, two patients presented with apparent severe exacerbation of MS symptoms several months after infusion of alemtuzumab; it could not be determined if these were MS relapses or manifestations of secondary autoimmunity directed toward the central nervous system. ${ }^{83}$ Both patients had continuing disease activity despite DMT use (dimethyl fumarate and fingolimod) prior to switching to alemtuzumab. The authors of the report considered these cases to be B-cell 
driven, in light of their successful resolution with plasma exchange plus administration of the B-cell-depleting antibody rituximab, and because the symptom onset coincided with post-alemtuzumab repopulation of B cells. ${ }^{83}$ Although the exact mechanism of alemtuzumab-associated autoimmune AEs has not been determined, it has been hypothesized that rapid hyper-repopulation of immature $B$ cells in the relative absence of regulatory T cells results in B-cell-driven autoimmunity. ${ }^{84}$ Further, secondary autoimmunity is known to occur in patients recovering from lymphopenia in other settings (eg, hematopoietic stem cell transplantation or anti-HIV therapy). ${ }^{85-88}$ Further work is needed to fully understand the biology underlying autoimmune AEs post-alemtuzumab.

\section{Malignancies}

Alemtuzumab may potentially be associated with an increased risk of some types of malignancies; however, the risk of all cancers combined was lower in the alemtuzumab RRMS clinical trial program than in the reference population. ${ }^{89}$ The most common malignancies were thyroid papillary carcinoma $(0.4 \%)$ and melanoma $(0.3 \%) .{ }^{89,90}$ As the risk management program included quarterly monitoring of TSH levels and patients with abnormal findings underwent further screening, the incidence of thyroid malignancies may have been inflated due to a more frequent assessment (ie, ascertainment bias). ${ }^{90}$

\section{Pregnancy}

As the effects of alemtuzumab treatment during pregnancy have not been determined, pregnant patients should not receive alemtuzumab treatment unless the likely benefit justifies the potential risk to the fetus. ${ }^{18}$ In the Phase II and III studies, pregnant or lactating patients were ineligible for treatment, but remained on study for safety follow-up. As of December 31, 2015, 200 pregnancies had occurred in 137 of 972 alemtuzumab-treated women in the clinical development program..$^{91}$ Of these pregnancies, 181 were complete and 11 were ongoing; outcomes of eight pregnancies were unknown. ${ }^{91}$ Among completed pregnancies with known outcomes, there were $122(67 \%)$ live births with no congenital abnormalities or birth defects; also, there were 39 (22\%) spontaneous abortions, $19(11 \%)$ elective abortions, and one $(0.6 \%)$ stillbirth. ${ }^{91}$ Elective abortions were due to personal choice $(n=6)$, extrauterine pregnancy $(n=3)$, anembryonic gestation $(n=2)$, or fetal defect ( $n=1$, cystic hygroma and hypoplastic heart); no information was available for the remaining seven cases. ${ }^{91}$

Women of child-bearing potential are advised to use effective contraception during alemtuzumab administration and for 4 months afterward. ${ }^{18}$ Transfer of TSH receptor antibodies from a mother with Graves' disease to her fetus can result in transient neonatal Graves' disease. One such case occurred in the CARE-MS studies, in which thyrotoxic crisis occurred in an infant born 1 year after alemtuzumab dosing in the mother. ${ }^{18,19}$ While quarterly TSH tests to evaluate thyroid function are recommended for all alemtuzumab-treated patients, experienced clinicians recommend monthly TSH testing in pregnant patients, for whom thyroid monitoring is especially important. ${ }^{92}$ Pregnancy outcomes in realworld patients with pregnancy onset within 4 months of alemtuzumab exposure are currently being collected in the Lemtrada Pregnancy Exposure Registry, a 19-country registry study. ${ }^{93}$

\section{Benefit:risk considerations for alemtuzumab treatment}

The decision to initiate alemtuzumab treatment should be weighed carefully for each patient. Many factors must be placed in perspective, including the risk of untreated or undertreated disease, as well as the overall benefit:risk profile. The extensive clinical development program established alemtuzumab as a highly effective DMT. ${ }^{11,12,36}$ Alemtuzumab is one of only a few MS DMTs for which approval was based on comparison to an active treatment, demonstrating significant reduction in relapses and improved disability outcomes, compared with SC IFNB-1a. The annualized relapse rate was reduced by $55 \%$ in CARE-MS I and $49 \%$ in CARE-MS II, compared with SC IFNB-1a. ${ }^{11,12}$ The risk of 6-month CDW was significantly reduced by $42 \%$ versus $\mathrm{SC}$ IFNB-1a in CARE-MS II. ${ }^{12}$ Efficacy was durable over at least 5 years in the CARE-MS studies in the absence of continuous treatment (ie, regular, repeated dosing). Furthermore, $68 \%$ (CARE-MS I) and 60\% (CARE-MS II) of patients did not receive alemtuzumab retreatment following their initial two courses, and 98\% and 92\%, respectively, did not receive another DMT after the initial two alemtuzumab courses. ${ }^{15,57}$ It is also noteworthy that these results were achieved with $96 \%$ of CARE-MS I patients and 91\% of CARE-MS II patients who entered the extension, remaining on study through 5 years. ${ }^{15,57}$ This high rate of patient retention rate contributes to the robustness of the data.

Data also support a consistent AE profile in patients with RRMS. AEs associated with alemtuzumab are well characterized, were consistent throughout the clinical development program, and responded to standard therapy. Risk mitigation strategies implemented in the alemtuzumab RRMS clinical development program allowed for early detection 
and treatment of AEs, and are the basis for practice recommendations detailed in alemtuzumab labeling. These risk mitigation measures, including monthly blood and urine tests and quarterly thyroid function tests that are continued for 48 months after the last alemtuzumab infusion, are now used in clinical practice. ${ }^{18,19}$ Patients must understand and accept the monitoring commitment before initiating treatment, including the need for continued monitoring even in the absence of alemtuzumab retreatment.

Most DMTs require continuous treatment, and studies have shown that patients can become fatigued by the need for frequent administration (especially parenteral administration) and cease to persist with therapy. ${ }^{29}$ The unique administration schedule of alemtuzumab (two courses 1 year apart) eliminates the need for continuous dosing, and thus lessens the potential for compliance issues.

Choice of therapy for patients with RRMS should weigh the importance of tailoring treatment to each patient's individual profile. In addition, a management plan should take account of the risks of leaving an MS patient without timely and appropriate treatment, as well as the risk of using inadequate agents, especially in the era of high-efficacy DMTs. Both CARE-MS I and the Phase II study showed that early in the disease course, treatment-naive RRMS patients with active disease who were treated with alemtuzumab were at lower risk for clinical and radiologic worsening, as well as for BVL, than those treated with SC IFNB-1a. ${ }^{11,36}$ The risk associated with a delay in using a high-efficacy DMT was confirmed in CARE-MS II, in which the clinical and radiologic benefits of alemtuzumab over SC IFNB-1a were also evident in RRMS patients with active disease who had been treated previously with a platform therapy. ${ }^{12}$ The results of CARE-MS I and II provide strong evidence in support of early alemtuzumab treatment in RRMS patients with active disease. Finally, the decision to prescribe a high-efficacy DMT should also consider the patient's risk tolerance and expectations for treatment. Many patients are willing to accept increased risk in exchange for increased efficacy, as demonstrated by a study in RRMS patients, which showed that risk tolerance increased with the degree of expected clinical benefit. ${ }^{94}$

Still, each patient must establish a personal threshold of acceptable risk. This threshold should be based on both the quantitative (eg, incidence) and qualitative (eg, discomfort, treatability) aspects of the AEs associated with treatment, on individual risk factors and the likely course of disease, and on the cumulative risk that may be associated with continuous treatment. It is important that clinicians take the time to educate patients regarding the potential benefits of treatment, predictable AEs, and the importance of commitment to the treatment decision, along with the monitoring requirements associated with each DMT, thus equipping patients to participate in the evaluation of therapy tradeoffs. The authors have found on occasion that after such a discussion, requesting patients to sign an informed consent form is helpful in clarifying benefit:risk. The risk mitigation strategies in place for alemtuzumab may assuage patients' and clinicians' concerns when linked to alemtuzumab's durable efficacy profile and abbreviated dosing regimen, compared with other DMTs.

\section{Conclusion}

Alemtuzumab is a high-efficacy DMT with demonstrated benefits in patients who are either treatment naive or who have had an inadequate response to prior therapy. Alemtuzumab has also demonstrated durable efficacy in the absence of continuous treatment, and the majority of RRMS patients did not require retreatment following the initial two courses through 5 years. Demonstrated benefits include a reduction in relapse rate, slowing or stabilization of the rate of disability worsening, improvement of disability in some patients, and a slowing of BVL. Safety data indicate that the risks associated with alemtuzumab are manageable with monitoring, and AEs respond to standard therapies. In alemtuzumab clinical trials, protocol-defined monitoring procedures detected IARs, infections, and autoimmune events, which allowed prompt successful management in the majority of cases. Overall, AEs decreased over time after alemtuzumab treatment, in contrast to experience with some other DMTs, which are associated with risks that persist or increase with chronic exposure. Data from postmarketing use have also provided valuable additional information regarding the safety profile of alemtuzumab. Through February 2017, 13,000 patients have been treated worldwide with alemtuzumab for MS. ${ }^{69}$ The postmarketing frequency of autoimmune AEs has generally been lower than expected based on the incidence observed in clinical trials. There is an increased frequency of infection and the potential for opportunistic infections following alemtuzumab treatment (most commonly, L. monocytogenes and CMV); however, there have been no medically confirmed reports of progressive multifocal leukoencephalopathy attributed to alemtuzumab to date. Ongoing evaluation of postmarketing safety reports continues to help refine the safety and benefit:risk profiles of alemtuzumab, as well as to identify potential new safety signals. Patient education, vigilance on the part of both 
physician and patient, and adherence to monthly laboratory monitoring are recommended to maintain the positive benefit:risk balance for alemtuzumab.

\section{Disclosure}

Editorial support was provided by Linda Wychowski, $\mathrm{PhD}$, and Rosemary Perkins, Envision Scientific Solutions. Funding for editorial support was provided by Sanofi. This manuscript was reviewed for scientific and medical accuracy by Darren P Baker, PhD, of Sanofi. JAC received personal compensation for consulting for Adamas, Celgene, Mallinckrodt, Merck, and Novartis and as a Co-Editor of Multiple Sclerosis Journal - Experimental, Translational and Clinical. EH received honoraria and grant support (Actelion, Biogen, Merck Serono, Novartis, Receptos, Roche, Sanofi, and Teva), and support from the Czech Ministry of Education research project Progres Q27/LF1. DH received speaker honoraria and consultant fees (Bayer, Biogen, Merck, Novartis, Roche, Sanofi, and Teva), financial support for research activities (Biogen), and support from the Czech Ministry of Education project Progres Q27/LF1. IK received speaker honoraria and consultant fees (Bayer, Biogen, Merck Serono, Novartis, and Teva), and financial support for research activities (Biogen). EM received speaker honoraria and consultant fees (Biogen, Merck Serono, Novartis, Sanofi, and Teva). The authors report no other conflicts of interest in this work.

\section{References}

1. Giovannoni G, Arnold D, Cohen J, et al. Long-term responders from the CARE-MS I study: no evidence of disease activity for 4 years following 2 courses of alemtuzumab and no further treatment. Neurology. 2016; 86(16 suppl):P3.054.

2. Hauser SL, Bar-Or A, Comi C, et al. Ocrelizumab versus interferon beta-1a in relapsing multiple sclerosis. $N$ Engl J Med. 2017;376: 221-234.

3. Ziemssen T, De Stefano N, Sormani MP, Van Wijmeersch B, Wiendl H, Kieseier BC. Optimizing therapy early in multiple sclerosis: an evidencebased view. Mult Scler Relat Disord. 2015;4(5):460-469.

4. University of California, San Francisco MS-EPIC Team: Cree BA, Gourraud PA, Oksenberg JR, et al. Long-term evolution of multiple sclerosis disability in the treatment era. Ann Neurol. 2016;80(4): 499-510.

5. Giovannoni G, Cook C, Rammohan K; CLARITY study group, et al. Sustained disease-activity-free status in patients with relapsing-remitting multiple sclerosis treated with cladribine tablets in the CLARITY study: a post-hoc and subgroup analysis. Lancet Neurol. 2011; 10(4):329-337.

6. Havrdova E, Galetta S, Hutchinson M, et al. Effect of natalizumab on clinical and radiological disease activity in multiple sclerosis: a retrospective analysis of the Natalizumab Safety and Efficacy in Relapsing-Remitting Multiple Sclerosis (AFFIRM) study. Lancet Neurol. 2009;8(3):254-260.

7. Rotstein DL, Healy BC, Malik MT, Chitnis T, Weiner HL. Evaluation of no evidence of disease activity in a 7-year longitudinal multiple sclerosis cohort. JAMA Neurol. 2015;72(2):152-158.
8. Stangel M, Penner I, Kallmann B, Lukas C, Kieseier BC. Towards the implementation of 'no evidence of disease activity' in multiple sclerosis treatment: the multiple sclerosis decision model. Ther $A d v$ Neurol Disord. 2015;8(1):3-13.

9. Beer S, Khan F, Kesselring J. Rehabilitation interventions in multiple sclerosis: an overview. J Neurol. 2012;259(9):1994-2008.

10. Tomassini V, Matthews PM, Thompson AJ, et al. Neuroplasticity and functional recovery in multiple sclerosis. Nat Rev Neurol. 2012; 8(11):635-646.

11. Cohen JA, Coles AJ, Arnold DL; CARE-MS I investigators, et al. Alemtuzumab versus interferon beta $1 \mathrm{a}$ as first-line treatment for patients with relapsing-remitting multiple sclerosis: a randomised controlled phase 3 trial. Lancet. 2012;380(9856):1819-1828.

12. Coles AJ, Twyman CL, Arnold DL; CARE-MS II investigators, et al. Alemtuzumab for patients with relapsing multiple sclerosis after disease-modifying therapy: a randomised controlled phase 3 trial. Lancet. 2012;380(9856):1829-1839.

13. Giovannoni G, Gold R, Kappos L, et al. Delayed-release dimethyl fumarate and disability assessed by the multiple sclerosis functional composite: integrated analysis of DEFINE and CONFIRM. Mult Scler J Exp Transl Clin. 2016;2:2055217316634111.

14. Prosperini L, Barletta V, De Giglio L, Fanelli F, Borriello G, Pozzilli C. A five-year clinical follow-up study of patients with multiple sclerosis who started natalizumab. Neurology. 2014;82(10 suppl):P7.215.

15. Coles A, Arnold DL, Cohen JA, et al. Patients with active RRMS and an inadequate response to prior therapy demonstrate durable improvements in relapse and disability following treatment with alemtuzumab: 5-year follow-up of the CARE-MS II study. Neurology. 2016; 86(16 suppl):P3.022.

16. Giovannoni G, Cohen JA, Coles AJ; CARE-MS II Investigators, et al. Alemtuzumab improves preexisting disability in active relapsingremitting MS patients. Neurology. 2016;87(19):1985-1992.

17. Biogen Inc. TYSABRI (natalizumab). Prescribing Information. Cambridge, MA: Biogen Idec; 2013.

18. Lemtrada. (alemtuzumab) Summary of Product Characteristics. 2016. Available from: http://www.ema.europa.eu/docs/en_GB/document_ library/EPAR_-_Product_Information/human/003718/WC500150521. pdf. Accessed March 29, 2017.

19. Lemtrada. (Alemtuzumab) Prescribing Information. Cambridge, MA: Genzyme Corporation; 2016.

20. Bezabeh S, Flowers CM, Kortepeter C, Avigan M. Clinically significant liver injury in patients treated with natalizumab. Aliment Pharmacol Ther. 2010;31(9):1028-1035.

21. Lisotti A, Azzaroli F, Brillanti S, Mazzella G. Severe acute autoimmune hepatitis after natalizumab treatment. Dig Liver Dis. 2012;44(4): 356-357.

22. Polman CH, O'Connor PW, Havrdova E; AFFIRM Investigators, et al. A randomized, placebo-controlled trial of natalizumab for relapsing multiple sclerosis. N Engl J Med. 2006;354(9):899-910.

23. Subei AM, Ontaneda D. Risk mitigation strategies for adverse reactions associated with the disease-modifying drugs in multiple sclerosis. CNS Drugs. 2015;29(9):759-771.

24. Filippi M, Rocca MA, Barkhof F; Attendees of the Correlation between Pathological MRI findings in MS workshop, et al. Association between pathological and MRI findings in multiple sclerosis. Lancet Neurol. 2012;11(4):349-360.

25. Kutzelnigg A, Lassmann H. Pathology of multiple sclerosis and related inflammatory demyelinating diseases. Handb Clin Neurol. 2014; 122(3):15-58.

26. Lublin FD, Baier M, Cutter G. Effect of relapses on development of residual deficit in multiple sclerosis. Neurology. 2003;61(11):1528-1532.

27. Multiple Sclerosis Therapy Consensus Group (MSTCG), Wiendl H, Toyka KV, et al. Basic and escalating immunomodulatory treatments in multiple sclerosis: current therapeutic recommendations. J Neurol. 2008;255(10):1449-1463.

28. Clerico M, Barbero P, Contessa G, Ferrero C, Durelli L. Adherence to interferon-beta treatment and results of therapy switching. J Neurol Sci. 2007;259(1-2):104-108. 
29. Cox D, Stone J. Managing self-injection difficulties in patients with relapsing-remitting multiple sclerosis. J Neurosci Nurs. 2006;38(3): $167-171$.

30. Costello K, Kennedy P, Scanzillo J. Recognizing nonadherence in patients with multiple sclerosis and maintaining treatment adherence in the long term. Medscape J Med. 2008;10(9):225.

31. Havrdova E, Arnold DL, Cohen JA; CARE-MS I and CAMMS03409 Investigators, et al. Alemtuzumab CARE-MS I 5-year follow-up: durable efficacy in the absence of continuous MS therapy. Neurology 2017;89(11):1107-1116.

32. Coles AJ, Cohen JA, Fox EJ; CARE-MS II and CAMMS03409 Investigators, et al. Alemtuzumab CARE-MS II 5-year follow-up: efficacy and safety findings. Neurology. 2017;89(11):1117-1126.

33. Coles AJ, Boyko AN, Cohen JA, et al. Alemtuzumab provides durable improvements in clinical outcomes in treatment-naive patients with active relapsing-remitting multiple sclerosis over 6 years in the absence of continuous treatment (CARE-MS I) (P213). Paper presented at: 32nd Congress of the European Committee for Treatment and Research in Multiple Sclerosis (ECTRIMS). September 14-17, 2016; London, UK.

34. Fox EJ, Alroughani R, Brassat D, et al. Efficacy of alemtuzumab is durable over 6 years in patients with active relapsing-remitting multiple sclerosis and an inadequate response to prior therapy in the absence of continuous treatment (CARE-MS II) (P1150). Paper presented at: 32nd Congress of the European Committee for Treatment and Research in Multiple Sclerosis (ECTRIMS). September 14-17, 2016; London, UK.

35. Ziemssen T, Thomas K. Alemtuzumab in the long-term treatment of relapsing-remitting multiple sclerosis: an update on the clinical trial evidence and data from the real world. Ther Adv Neurol Disord. Prepublished August 4, 2017, DOI: 10.1177/1756285617722706.

36. CAMMS223 Trial Investigators, Coles AJ, Compston DA, et al. Alemtuzumab vs. interferon beta-1a in early multiple sclerosis. $N$ Engl J Med. 2008;359(17):1786-1801.

37. Kovarova I, Arnold DL, Cohen JA, et al. Alemtuzumab pharmacokinetics and pharmacodynamics in Comparison of Alemtuzumab and Rebif ${ }^{\sqrt{ }}$ Efficacy in Multiple Sclerosis I (P341). Paper presented at: European Neurological Society; June 9-12, 2012; Prague, Czech Republic.

38. Fletcher JM, Lalor SJ, Sweeney CM, Tubridy N, Mills KHG. T cells in multiple sclerosis and experimental autoimmune encephalomyelitis. Clin Exp Immunol. 2010;162(1):1-11.

39. Zhang X, Tao Y, Chopra M, et al. Differential reconstitution of T cell subsets following immunodepleting treatment with alemtuzumab (anti-CD52 monoclonal antibody) in patients with relapsing-remitting multiple sclerosis. J Immunol. 2013;191(12):5867-5874.

40. Zozulya AL, Wiendl H. The role of regulatory $\mathrm{T}$ cells in multiple sclerosis. Nat Clin Pract Neurol. 2008;4(7):384-398.

41. Cross AH, Waubant E. MS and the B cell controversy. Biochim Biophys Acta. 2011;1812(2):231-238.

42. Kasper LH, Arnold DL, Cohen JA, et al. Lymphocyte subset dynamics following alemtuzumab treatment in the CARE-MS II study. Mult Scler. 2013;19(11 suppl):P531.

43. Fox EJ. Alemtuzumab in the treatment of relapsing-remitting multiple sclerosis. Expert Rev Neurother. 2010;10(12):1789-1797.

44. Freedman MS, Kaplan JM, Markovic-Plese S. Insights into the mechanisms of the therapeutic efficacy of alemtuzumab in multiple sclerosis J Clin Cell Immunol. 2013;4(4):1000152.

45. Turner MJ, Lamorte MJ, Chretien N, et al. Immune status following alemtuzumab treatment in human CD52 transgenic mice. J Neuroimmunol. 2013;261(1-2):29-36.

46. Jones JL, Anderson JM, Phuah CL, et al. Improvement in disability after alemtuzumab treatment of multiple sclerosis is associated with neuroprotective autoimmunity. Brain. 2010;133(Pt 8):2232-2247.

47. Dombrowski Y, O’Hagan T, Dittmer M, et al. Regulatory T cells promote myelin regeneration in the central nervous system. Nat Neurosci. 2017;20(5):674-680.

48. Clark RA, Watanabe R, Teague JE, et al. Skin effector memory T cells do not recirculate and provide immune protection in alemtuzumabtreated CTCL patients. Sci Transl Med. 2012;4(117):117ra7.
49. Coles AJ, Wing M, Smith S, et al. Pulsed monoclonal antibody treatment and autoimmune thyroid disease in multiple sclerosis. Lancet. 1999;354(9191):1691-1695.

50. Hu Y, Turner MJ, Shields J, et al. Investigation of the mechanism of action of alemtuzumab in a human CD52 transgenic mouse model. Immunology. 2009;128(2):260-270.

51. Margolin D, Kieseier B, Palmer J, Ziemssen T. Alemtuzumab has similar efficacy and safety in active relapsing-remitting multiple sclerosis (RRMS) patients who were treatment-naive or who relapsed on prior therapy (P2.209). Neurology. 2014;82(10 suppl):P2.209.

52. Arnold DL, Traboulsee A, Cohen JA, et al. Alemtuzumab demonstrates durable reduction of MRI activity over 5 years in CARE-MS I with the majority of patients treatment-free for 4 years (P1100). Paper presented at: 31 st Congress of the European Committee for Treatment and Research in Multiple Sclerosis (ECTRIMS); October 7-10, 2015; Barcelona, Spain.

53. Barkhof F, Cohen JA, Coles AJ, et al. Alemtuzumab slows brain volume loss over 5 years in patients with active relapsing-remitting multiple sclerosis with most patients not receiving treatment for 4 years: CARE-MS I and II extension study. Paper presented at: 31st Congress of the European Committee for Treatment and Research in Multiple Sclerosis (ECTRIMS); October 7-10, 2015; Barcelona, Spain.

54. Fox EJ, Arnold DL, Cohen JA, et al. Durable efficacy of alemtuzumab on clinical outcomes over 5 years in CARE-MS II with most patients free from treatment for 4 years (P1102). Paper presented at: 31st Congress of the European Committee for Treatment and Research in Multiple Sclerosis (ECTRIMS); October 7-10, 2015; Barcelona, Spain.

55. Havrdova E, Arnold DL, Cohen JA, et al. Durable efficacy of alemtuzumab on clinical outcomes over 5 years in treatment-naive patients with active relapsing-remitting multiple sclerosis with most patients not receiving treatment for 4 years: CARE-MS I extension study (P152). Paper presented at: 31st Congress of the European Committee for Treatment and Research in Multiple Sclerosis (ECTRIMS); October 7-10, 2015; Barcelona, Spain.

56. Traboulsee A, Cohen JA, Coles AJ, et al. Alemtuzumab demonstrates durable reduction of MRI activity over 5 years in CARE-MS I with the majority of patients treatment-free for 4 years (P1103). Paper presented at: 31 st Congress of the European Committee for Treatment and Research in Multiple Sclerosis (ECTRIMS) 2015; Barcelona, Spain.

57. Limmroth V, Giovannoni G, Arnold DL, et al. Treatment-naive patients with active RRMS demonstrate durable improvements in relapse and disability following treatment with alemtuzumab: 5-year follow-up of the CARE-MS I Study. Neurology. 2016;86(16 suppl):S51.004.

58. Fox EJ, Arnold DL, Cohen JA, et al. Durable efficacy in RRMS patients receiving two annual courses of alemtuzumab and no additional treatment for 4 years: pooled analysis of CARE-MS I and II. Neurology. 2016;86(16 suppl):S51.005.

59. Arroyo Gonzalez R, Kita M, Crayton H; CARE-MS I and II Investigators, et al. Alemtuzumab improves quality-of-life outcomes compared with subcutaneous interferon beta-1a in patients with active relapsing-remitting multiple sclerosis. Mult Scler. 2017;23(10): 1367-1376.

60. Arroyo González R, Moreau T, Hartung HP, et al. Sustained reduction in disability with alemtuzumab is associated with durable quality-oflife improvement on SF-36 over 4 years in CARE-MS II patients with RRMS though most were treatment-free after year 1 (P1152). Paper presented at: 31 st Congress of the European Committee for Treatment and Research in Multiple Sclerosis (ECTRIMS); October 7-10, 2015; Barcelona, Spain.

61. Kita M, Cohen J, Moreau TT, et al. Improvement in disability with alemtuzumab is associated with quality of life improvement over 3 years in patients who relapsed on prior therapy. Neurology. 2015; 84(14 suppl):P3.260.

62. Mayer L, Fox EJ, LaGanke C, et al. Incidence of infusion-associated reactions decreases with subsequent courses of alemtuzumab: 5-year data from the CARE-MS extension study (DX44). Paper presented at: 30th Annual Meeting of the Consortium of Multiple Sclerosis Centers (CMSC); June 1-4, 2016; National Harbor, MD, USA. 
63. Breslin S. Cytokine-release syndrome: overview and nursing implications. Clin J Oncol Nurs. 2007;11(1 Suppl):37-42.

64. Maggi E, Vultaggio A, Matucci A. Acute infusion reactions induced by monoclonal antibody therapy. Expert Rev Clin Immunol. 2011; 7(1):55-63.

65. McEwan L, Caon C, Chieffe C, Mayer L, Saldana-King T, Miller CE. Best practices in alemtuzumab administration: practical recommendations for infusion patients with multple sclerosis. J Infus Nurs. 2016; 39(2):93-104.

66. Caon C, Namey M, Meyer C, et al. Prevention and management of infusion-associated reactions in the comparison of Alemtuzumab and Rebif ${ }^{\circledR}$ Efficacy in Multiple Sclerosis (CARE-MS) Program. Int $J$ MS Care. 2015;17(4):191-198.

67. Wray S, Boyko AN, Braley T, et al. Administration of alemtuzumab on nonconsecutive days does not impact infusion-associated reactions, efficacy, or lymphocyte depletion. Neurology. 2015;84(14 suppl): P7.277.

68. Jung Henson L, Arnold D, Cohen J, et al. Incidence of infection decreases over time in alemtuzumab-treated patients with relapsingremitting multiple sclerosis: 4-year follow-up of the CARE-MS Studies. Neurology. 2015;84(14 suppl):P7.265.

69. Selmaj KW, Habek M, Bass A, et al. Efficacy and safety of alemtuzumab in patients with RRMS is durable over 10 years: follow-up from the CAMMS223 study. Neurology. 2017;88(16 suppl):P5.338.

70. Holmøy T, von der Lippe H, Leegaard TM. Listeria monocytogenes infection associated with alemtuzumab a case for better preventive strategies. BMC Neurol. 2017;17(1):65.

71. Décard BF, Thöne J, Haghikia A, et al. Listeria rhombencephalitis mimicking a demyelinating event in an immunocompetent young patient. Mult Scler. 2017;23(1):123-125.

72. Hernandez-Milian A, Payeras-Cifre A. What is new in listeriosis? Biomed Res Int. 2014;2014:358051.

73. Clerico M, De Mercanti S, Artusi CA, Durelli L, Naismith RT. Active CMV infection in two patients with multiple sclerosis treated with alemtuzumab. Mult Scler. 2017;23(6):874-876.

74. Pfeuffer S, Beuker C, Ruck T, et al. Acute cholecystitis during treatment with alemtuzumab in 3 patients with RRMS. Neurology. 2016; 87(22):2380-2381.

75. Sheikh-Taha M, Corman LC. Pulmonary Nocardia beijingensis infection associated with the use of alemtuzumab in a patient with multiple sclerosis. Mult Scler. 2017;23(6):872-874.

76. Senior PA, Arnold DL, Cohen JA, et al. Incidence and timing of thyroid adverse events in patients with RRMS treated with alemtuzumab through 5 years of the CARE-MS Studies. Neurology. 2016; 86(16 suppl):P2.086.

77. Cuker A, Arnold DL, Cohen JA, et al. Detection and management of immune thrombocytopenia in alemtuzumab-treated patients in the Multiple Sclerosis Clinical Development Program (P590). Paper presented at: 31 st Congress of the European Committee for Treatment and Research in Multiple Sclerosis (ECTRIMS); October 7-10, 2015; Barcelona, Spain.

78. Cuker A, Coles AJ, Sullivan H, et al. A distinctive form of immune thrombocytopenia in a phase 2 study of alemtuzumab for the treatment of relapsing-remitting multiple sclerosis. Blood. 2011;118(24): 6299-6305.

79. Ghodasara RS, Rarick MB, Mosley MC, et al. Alemtuzumab causes significant, transient, post-infusion thrombocytopenia and other non autoimmune cytopenias following initial and subsequent courses. Paper presented at: Consortium of Multiple Sclerosis Centers Annual Meeting; June 1-4 2016; National Harbor, MD, USA.
80. Ranganathan U, Kaunzner U, Foster S, Vartanian T, Perumal JS. Immediate transient thrombocytopenia at the time of alemtuzumab infusion in multiple sclerosis. Multiple Scler. Epub 2017 Mar 1.

81. Rodeghiero F, Stasi R, Gernsheimer T, et al. Standardization of terminology, definitions and outcome criteria in immune thrombocytopenic purpura of adults and children: report from an international working group. Blood. 2009;113(11):2386-2393.

82. Wynn D, Arnold DL, Cohen JA, et al. Detection, incidence, and management of glomerulonephritis in the alemtuzumab clinical development program (P597). Paper presented at: 29th Congress of the European Committee for Treatment and Research in Multiple Sclerosis (ECTRIMS); October 2-5, 2013; Copenhagen, Denmark.

83. Haghikia A, Dendrou CA, Schneider R, et al. Severe B-cell-mediated CNS disease secondary to alemtuzumab therapy. Lancet Neurol. 2017; 16(3):104-106.

84. Baker D, Herrod SS, Alvarez-Gonzalez C, Giovannoni G, Schmierer K. Interpreting lymphocyte reconstitution data from the pivotal phase 3 trials of alemtuzumab. JAMA Neurol. 2017;74(8):961-969.

85. Chen F, Day SL, Metcalfe RA, et al. Characteristics of autoimmune thyroid disease occurring as a late complication of immune reconstitution in patients with advanced human immunodeficiency virus (HIV) disease. Medicine (Baltimore). 2005;84(2):98-106.

86. Daikeler T, Tyndall A. Autoimmunity following haematopoietic stem-cell transplantation. Best Pract Res Clin Haematol. 2007;20(2): 349-360.

87. Jubault V, Penfornis A, Schillo F, et al. Sequential occurrence of thyroid autoantibodies and Graves' disease after immune restoration in severely immunocompromised human immunodeficiency virus-1infected patients. J Clin Endocrinol Metab. 2000;85(11):4254-4257.

88. Ting SS, Ziegler JB, Vowels MR. Acquired autoimmune thrombocytopenia post-bone marrow transplantation for severe combined immunodeficiency. Bone Marrow Transplant. 1998;21(8):841-843.

89. Miller T, Habek M, Coles AJ, et al. Analysis of data from RRMS alemtuzumab-treated patients in the clinical program to evaluate incidence rates of malignancy. Paper presented at: Joint Americas Committee for Treatment and Research in Multiple Sclerosis (ACTRIMS) European Committee for Treatment and Research in Multiple Sclerosis (ECTRIMS) Meeting; September 10-13, 2014; Boston, MA, USA.

90. Lecumberri B, Arnold DL, Cohen JA, et al. Detection of thyroid malignancies in alemtuzumab-treated patients in the multiple sclerosis clinical development program. Mult Scler. 2015;21(S11):P1117.

91. Oh J, Achiron A, Chambers C, et al. Pregnancy outcomes in patients with RRMS who received alemtuzumab in the Clinical Development Program (S24.008). Neurology. 2016;86(16 Suppl):S24.008.

92. Hassoun L, Eisele J, Thomas K, Ziemssen T. Hands on alemtuzumab experience from clinical practice: whom and how to treat. Multi Scler Demyelinating Disorders. 2016;1(10):1-14.

93. Rog D, Seferta N, Daniel D, et al. LEMTRADA ${ }^{\circledR}$ (Alemtuzumab) Pregnancy Exposure Registry: study design and enrolment procedures for pregnant women with multiple sclerosis exposed to alemtuzumab. MS Trust 2015 Conference, November 8-10, 2015. Windsor, UK.

94. Johnson FR, Van Houtven G, Ozdemir S, et al. Multiple sclerosis patients' benefit-risk preferences: serious adverse event risks versus treatment efficacy. J Neurol. 2009;256(4):554-562.

95. Mayer L, Casady L, Clayton G, et al. Management of infusion-associated reactions in alemtuzumab-treated relapsing-remitting multiple sclerosis patients (P880). Paper presented at: Joint Americas Committee for Treatment and Research in Multiple Sclerosis (ACTRIMS) European Committee for Treatment and Research in Multiple Sclerosis (ECTRIMS) Meeting; September 10-13, 2014; Boston, MA, USA. 
Therapeutics and Clinical Risk Management

Dovepress

\section{Publish your work in this journal}

Therapeutics and Clinical Risk Management is an international, peerreviewed journal of clinical therapeutics and risk management, focusing on concise rapid reporting of clinical studies in all therapeutic areas outcomes, safety, and programs for the effective, safe, and sustained use of medicines. This journal is indexed on PubMed Central, CAS,
EMBase, Scopus and the Elsevier Bibliographic databases. The manuscript management system is completely online and includes a very quick and fair peer-review system, which is all easy to use. Visit http://www.dovepress.com/testimonials.php to read real quotes from published authors.

Submit your manuscript here: http://www.dovepress.com/therapeutics-and-clinical-risk-management-journal 